\title{
Protein Synthesis-Dependent Associative Long-Term Memory in Larval Zebrafish
}

\author{
Flora I. Hinz, ${ }^{1,2}$ Mark Aizenberg, ${ }^{1,2}$ Georgi Tushev, ${ }^{2}$ and Erin M. Schuman ${ }^{2}$ \\ ${ }^{1}$ Division of Biology, California Institute of Technology, Pasadena, California 91125, and ${ }^{2}$ Max Planck Institute for Brain Research, D-60528, Frankfurt am \\ Main, Germany
}

The larval zebrafish is a model organism to study the neural circuitry underlying behavior. There exist, however, few examples of robust long-term memory. Here we describe a simple, unrestrained associative place-conditioning paradigm. We show that visual access to a group of conspecifics has rewarding properties for 6- to 8-day-old larval zebrafish. We use this social reward as an unconditioned stimulus and pair it with a distinct visual environment. After training, larvae exhibited spatial preference for the location previously paired with the social reward for up to $36 \mathrm{~h}$, indicating that zebrafish larvae can exhibit long-term associative memory. Furthermore, incubation with a protein synthesis inhibitor or an NMDAR-antagonist impaired memory. In future experiments, this learning paradigm could be used to study the social interactions of larval zebrafish or paired with cell-specific metabolic labeling to visualize circuits underlying memory formation.

\section{Introduction}

Long-term memory formation has been shown to be protein synthesis-dependent in a number of different organisms, including teleost fish (Agranoff and Klinger, 1964; Davis and Squire, 1984). Although the larval zebrafish has become a prominent model organism for studying neural circuitry underlying behavior (e.g., Wyart et al., 2009; Del Bene et al., 2010; Fetcho and McLean, 2010), there are few robust, protein synthesisdependent learning paradigms. A number of associative conditioning paradigms have recently been developed for adult zebrafish (Blank et al., 2009; Braubach et al., 2009; Mathur et al., 2011), but currently only nonassociative paradigms (Best et al., 2008; Roberts et al., 2011) or aversive associative paradigms (Lee et al., 2010; Aizenberg and Schuman, 2011) that result in shortterm memory exist for larval zebrafish. Furthermore, the aversive conditioning paradigms train individual larvae, making them difficult for high-throughput screens or proteomics approaches. Recently, Wolman et al. (2011) have shown that spaced training blocks of repetitive visual stimuli elicit protein synthesisdependent long-term habituation (nonassociative learning) in larval zebrafish lasting up to $24 \mathrm{~h}$, which is disrupted by cycloheximide incubation during training.

Here, we describe the first associative conditioning paradigm that induces long-term memory formation in larval zebrafish.

\footnotetext{
Received Feb. 6, 2013; revised Aug. 9, 2013; accepted Aug. 10, 2013.

Author contributions: F.I.H., M.A., and E.M.S. designed research; F.I.H. and M.A. performed research; G.T. contributed unpublished reagents/analytic tools; F.I.H. analyzed data; F.I.H. and E.M.S. wrote the paper.

This work was supported by the Max Planck Society. We thank the Max Planck Institute for Brain Research workshop for their help in constructing the chambers and behavioral box and Dr. Susanne tom Dieck and Dr. Cyril Hanus for their helpful critique of the manuscript.

The authors declare no competing financial interests.

Correspondence should be addressed to Dr. Erin M. Schuman, Max Planck Institute for Brain Research, Max-vonLaue-Str. 4, D-60528, Frankfurt am Main, Germany. E-mail: erin.schuman@brain.mpg.de.

DOI:10.1523/JNEUROSCI.0560-13.2013

Copyright $\odot 2013$ the authors $\quad 0270-6474 / 13 / 3315382-06 \$ 15.00 / 0$
}

Using a custom-built conditioning chamber, we show that visual access to a group of conspecifics has rewarding properties for 6 to 8-day-old larval zebrafish, as previously described for adult zebrafish (Al-Imari and Gerlai, 2008; Gómez-Laplaza and Gerlai, 2010; Sison and Gerlai, 2011). We then use this social reward as an unconditioned stimulus and pair it with a distinct visual environment during training. After training, larvae exhibited a preference for the visually demarcated area of the chamber previously paired with the social reward for up to $36 \mathrm{~h}$, demonstrating longterm associative learning. Furthermore, incubation with the protein synthesis inhibitors (PSIs) or an NMDAR antagonist during training impaired memory retention, demonstrating that the associative learning is protein synthesis- and NMDAR-dependent. In future experiments, this learning paradigm can be used to study social interactions of larval zebrafish or paired with cellspecific metabolic labeling to visualize circuits underlying memory formation.

\section{Materials and Methods}

Zebrafish stocks and husbandry. AB wild-type adult fish strains were kept at $28^{\circ} \mathrm{C}$ on a $14 \mathrm{~h} \mathrm{light} / 10 \mathrm{~h}$ dark cycle. Embryos of either sex were obtained from natural spawnings and were maintained in $\mathrm{E} 3$ embryo medium ( $5 \mathrm{~mm} \mathrm{NaCl}, 0.17 \mathrm{~mm} \mathrm{KCl}, 0.33 \mathrm{~mm} \mathrm{CaCl}_{2}, 0.33 \mathrm{~mm} \mathrm{MgSO}_{4}$ ) at $28^{\circ} \mathrm{C}$ on a $14 \mathrm{~h}$ light $/ 10 \mathrm{~h}$ dark cycle. Larvae were not fed for the duration of the experiment.

Behavioral chambers. The testing and training chambers were custom constructed from white plastic and transparent Plexiglas. Both chambers were $12.7 \mathrm{~cm} \times 8.5 \mathrm{~cm}$ and $1 \mathrm{~cm}$ deep, divided into 14 individual channels by removable partitions, which were slanted at an increasing angle from the middle outwards to prevent the creation of blind spots during video monitoring (Fig. 1a). The bottom of the training and testing chamber was made of transparent Plexiglas, whereas the sides were made of white plastic. The testing and training chambers were identical, except that the testing chamber had completely opaque partitions made of single pieces of white plastic, whereas the training chamber had partitions that were half-transparent Plexiglas and half-opaque white plastic. The be- 
a

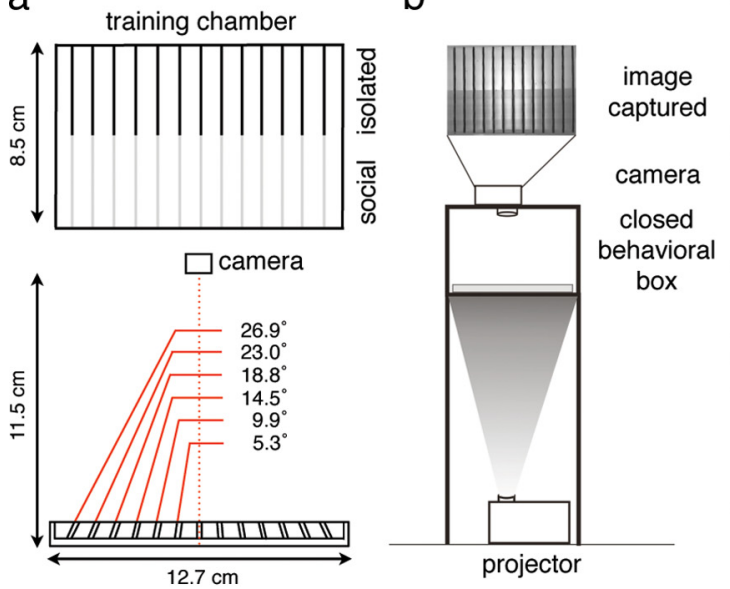

d

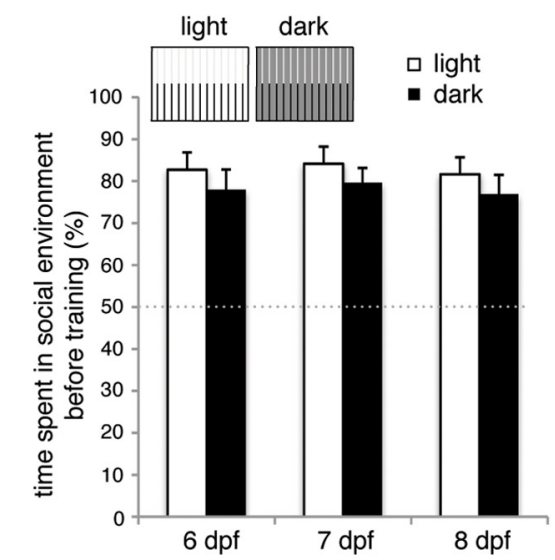

C

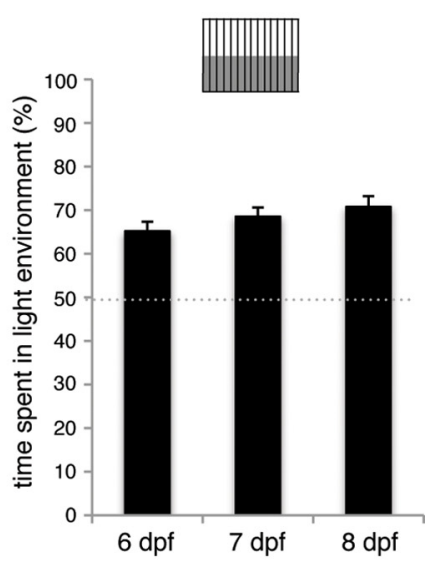

e

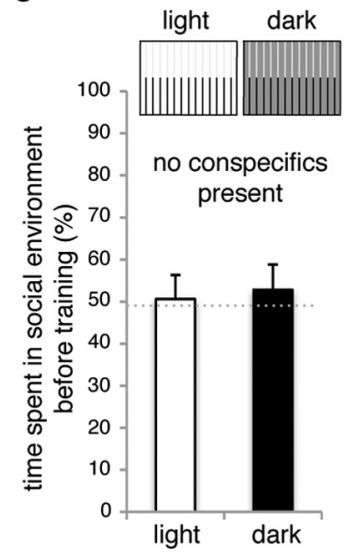

Figure 1. Unconditioned light and social preference. $\boldsymbol{a}$, Schematic of training chamber. The training chamber consists of 14 individual channels, separated on one side by opaque barriers, creating an isolated environment, and on the other side by clear barriers, creating a social environment in which larvae can see their conspecifics. Barriers are slanted at increasing angles toward the outer edges of the testing and training chambers to prevent occlusion of the larvae by the barriers from the camera, situated $11.5 \mathrm{~cm}$ above. $\boldsymbol{b}$, The testing and training chambers were placed in a custom-built, enclosed behavioral box that isolated the larvae from any outside visual or acoustic stimuli. The behavioral box had a semitransparent bottom, onto which different light environments were projected using a computer-controlled projector, while the position of the larvae was captured every 10 s using a camera mounted above. c, Percentage of time spent in light environment during $15 \mathrm{~min}$ period by larval zebrafish. Under conditions tested here, larvae showed moderate preference for light, which increases slightly, though not significantly $(6 \mathrm{dpf}$ vs 8 $\mathrm{dpf}, p=0.091$ ), with age. $n=120-168$ per condition. Error bars indicate SEM. $\boldsymbol{d}$, Percentage of time spent in social environment during $15 \mathrm{~min}$ period by larval zebrafish, in both light and dark illumination conditions. Differences between age groups were not statistically significant. $n=27$ or 28 per condition.e, Percentage of time spent in social environment during 15 min period by larval zebrafish placed into the training chamber individually, without conspecifics in adjacent channels, in both light and dark conditions. $n=28$ per condition.

havioral box measured $23 \mathrm{~cm}$ in total height, whereas the enclosed compartment was $11 \mathrm{~cm} \times 16 \mathrm{~cm} \times 13 \mathrm{~cm}$. The bottom was made of Plexiglas covered with a thin layer of semitransparent soft plastic. The rest of the behavioral box was constructed of white plastic. The front of the behavioral box had a sliding door and the top had a hole to allow visual access.

Behavioral assay, video recording, and behavioral analysis. The testing chamber (Fig. 1a) was filled with $\sim 30 \mathrm{ml}$ of E3 embryo medium and 6-8 dpf larval zebrafish that showed high exploratory behavior (swam across Petri dish instead of remaining at the walls, swam near the surface indicating that swim bladders were fully inflated and avoided capture) were placed individually into the 12 center channels. The larvae were given $\sim 5$ min to acclimate before the testing chamber was placed in the behavioral box (Fig. 1b). To monitor light environment preference, the projector (Optoma Pico Pocket DLP Projector, model PK301) was controlled using MATLAB (MathWorks) to illuminate the behavioral box, creating two different but equally sized light environments. The dark environment was created by setting the projector to emit red, green, blue values of 120 , whereas the light environment was created by the projector emitting red, green, blue values of 230 . This corresponds to $65 \pm 6.32 \mathrm{~cd} / \mathrm{m}^{2}$ and $279.5 \pm 32.55$ $\mathrm{cd} / \mathrm{m}^{2}$, respectively, as measured with a Konica Minolta LM-1 Luminance meter. The testing chamber was oriented such that the light and dark environments met at the midline of the testing chamber. Larval zebrafish position was captured using a Phillips webcam (SPC 2050NC) every $10 \mathrm{~s}$ for a period of $15 \mathrm{~min}$.

During Test 1, in which we quantified the unconditioned light environment preference, the position of individual larval zebrafish in the testing chamber was monitored for two $15 \mathrm{~min}$ periods. In between these two periods, the orientation of the light environments projected onto the behavioral box was rotated by $180^{\circ}$, but the orientation of the testing chamber within the behavioral box remained constant. Test 1 began immediately after the testing chamber was placed inside the behavioral box, by establishing light and dark regions. The larval zebrafish position was first captured $10 \mathrm{~s}$ after onset of Test 1 . For training, the training chamber was filled with E3 embryo medium. Larvae previously in the testing chamber were pipetted into the training chamber in the same order. The training chamber was carefully placed into the behavioral box. The same light environments as previously described were projected onto the behavioral box, and the training chamber was oriented such that the social environment (with clear barriers) was paired with the dark environment. The orientation of light environment and the training chamber were rotated by $180^{\circ}$ every $45 \mathrm{~min}$. Training consisted of four $45 \mathrm{~min}$ periods, for a total of $3 \mathrm{~h}$. Larval position was not monitored during training. Control group larvae were gently removed from the testing chamber after Test 1 , immediately returned to the same channel in the testing chamber and exposed to the same training light environment conditions as the experimental group.

After training, larvae were individually housed in a 12-well plate containing $1.5 \mathrm{ml} \mathrm{E3}$ embryo medium per well. The plate was marked to keep track of each individual larva and was incubated at $28^{\circ} \mathrm{C}$ on a $14 \mathrm{~h}$ light $/ 10 \mathrm{~h}$ dark cycle for $\sim 14 \mathrm{~h}$. Next, larvae were returned to the testing chamber in the same order as before to quantify light environment preference of individual larvae after conditioning. As above, larvae were allowed to acclimate to the testing chamber for 5 min before being placed into the behavioral box. Test 2 began immediately after the testing chamber was placed inside the behavioral box, by establishing light and dark regions. The larval zebrafish position was first captured $10 \mathrm{~s}$ after onset of Test 2 . Test 2 consisted of two 15 min periods during which the light and dark environments were projected onto the bottom of the behavioral box while the position of the larvae was monitored from above.

Each frame of larvae position taken during Test 1 and Test 2 was saved automatically and scored manually. Larvae detected in the light environment were scored as +1 , whereas larvae detected in the dark environment were scored at 0 . If larvae position could not be identified, a score of 0.5 was recorded for that frame; $0.13 \%$ of frames were scored as 0.5 . Scores for all 90 frames (taken every $10 \mathrm{~s}$ for $15 \mathrm{~min}$ ) were added, and percentage of time spent in the light environment was calculated. Preference difference scores, as a measure of memory retention, were calcu- 
a



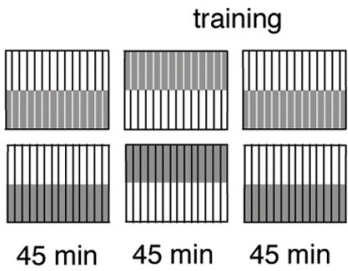

test 1

b

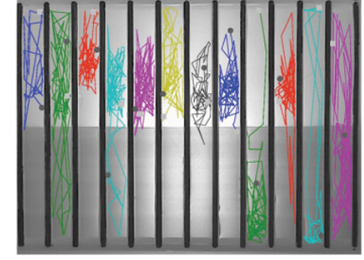

C

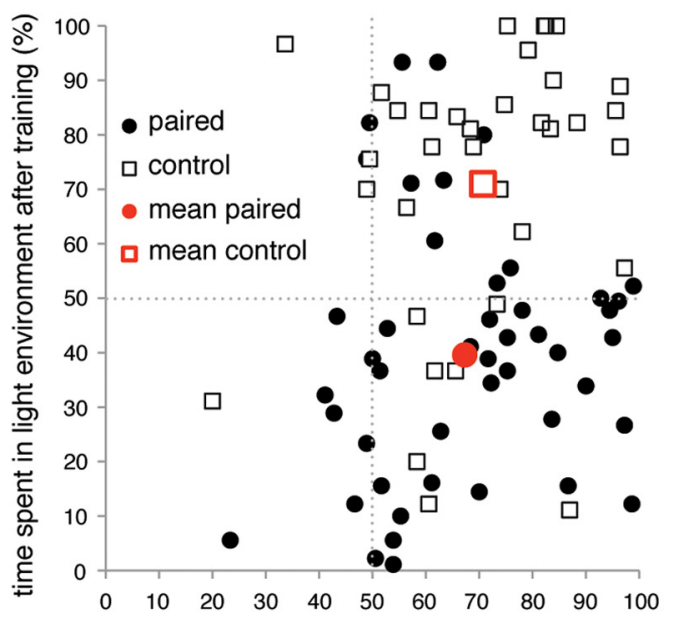

time spent in light environment before training (\%)

e

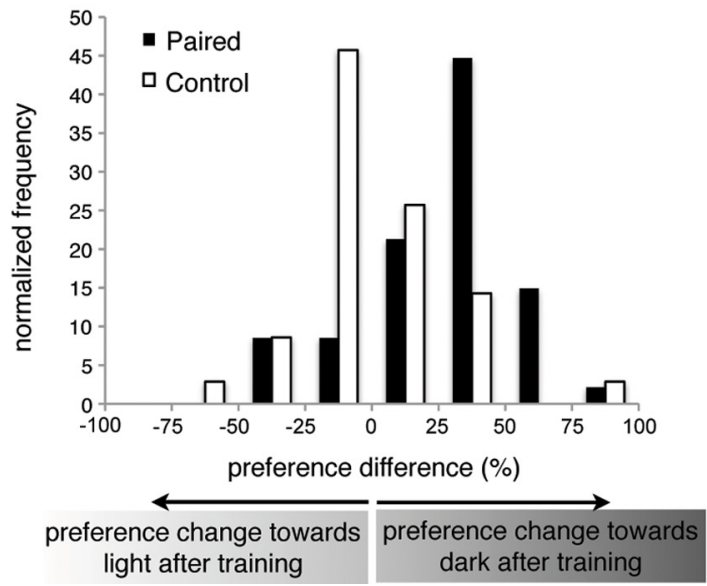

test 2

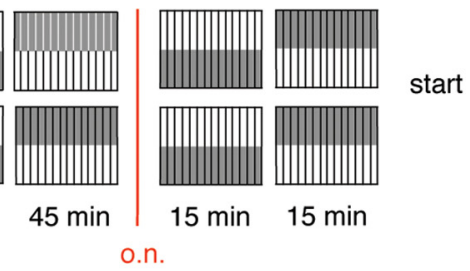

test 2

light preference after training

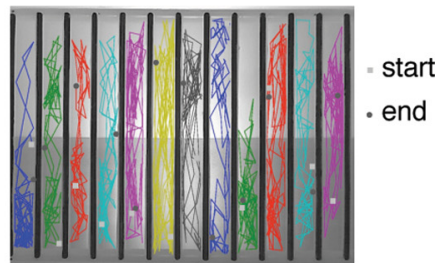

d

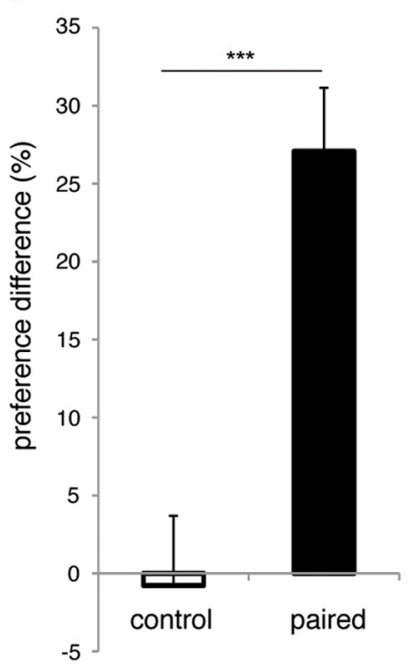

f

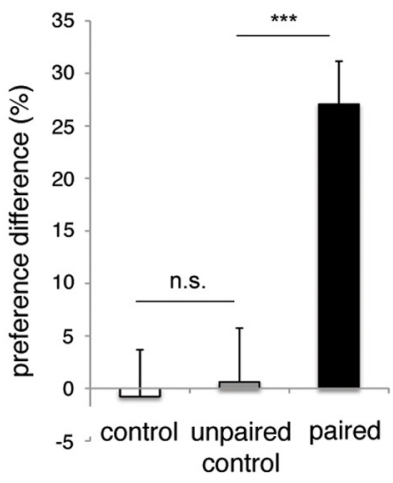

Figure 2. Associative place-conditioning in larval zebrafish. $a$, Associative place-conditioning paradigm. Unconditioned light preference was determined during Test 1 , followed by a $3 \mathrm{~h}$ training period during which dark and social environment were paired. Light preference after conditioning was determined during Test 2, $14 \mathrm{~h}$ after training. Control fish were exposed to the same pattern of light and dark environments but no social environment. $\boldsymbol{b}$, Sample position traces of individual larval zebrafish during Test 1, showing unconditioned light preference, and Test 2, $14 \mathrm{~h}$ after training, showing conditioned place preference. Light and gray squares represent position of individual larvae in the first and last frames, respectively. $c$, Percentage of time spent in the light environment before ( $x$-axis) and after ( $y$-axis) training of unpaired (black) and control (white) larvae. Red markers represent mean light preference. $\boldsymbol{d}$, The preference difference (\%) of experimental (black) and control (white) groups; positive values indicate a relative preference for the dark area of the chamber after training. Error bars indicate SEM. ${ }^{* * *} p<0.001$ and Cohen's $d \geq 0.8$. $n=$ 35-47 per condition. $\boldsymbol{e}$, Preference difference distribution of experimental (black) and control (white) groups. $\boldsymbol{f}$, Light preference difference of experimental (black), control (white), and unpaired control (gray). Error bars indicate SEM. ${ }^{* * *} p<0.001$ and Cohen's $d \geq 0.8 . n=35-48$ per condition. n.s., Not significant; 0.n., overnight. 
a

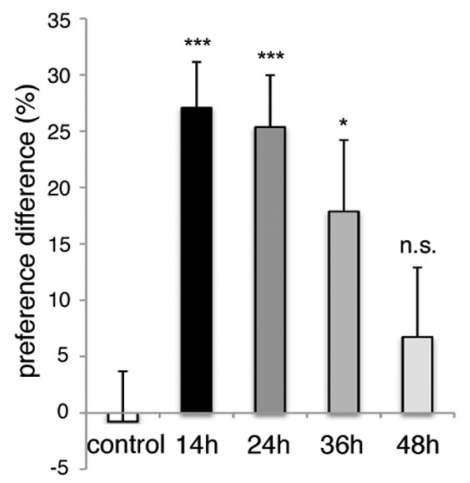

b

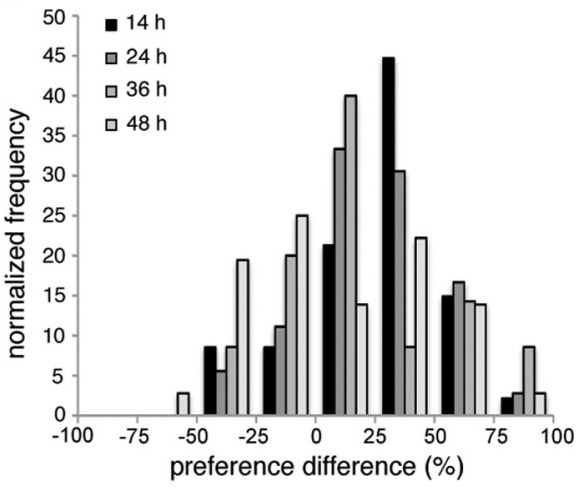

Figure 3. Memory is persistent. To test memory retention, the time between training and Test 2 was increased to up to $48 \mathrm{~h} . \boldsymbol{a}$ Light preference difference of larvae tested $14-48 \mathrm{~h}$ (black to light gray) after training and control (white) larvae; positive values indicate a preference for the dark area of the chamber. Error bars indicate SEM. ${ }^{* * *} p<0.001$ and Cohen's $d \geq 0.8$. $^{* *} p<0.01$ and Cohen's $d \geq 0.6$. ${ }^{*} p<0.05$ and Cohen's $d \geq 0.45 . n=35-47$ per condition. n.s., Not significant. $\boldsymbol{b}$, Preference difference distribution of larvae tested $14-48 \mathrm{~h}$ (black to light gray).

lated by subtracting percentage of time spent in light environment for the first period of Test 2 from the mean percentage of time spent in light environment for Test 1. Significance was calculated using paired and unpaired two-tailed $t$ tests. All error bars represent SEM. Effect size was calculated using Cohen's d test.

Memory retention was investigated by prolonging the time period between training and Test 2 . As described before, larvae were placed individually in 12 -well plates at $28^{\circ} \mathrm{C}$ on a $14 \mathrm{~h}$ light $/ 10 \mathrm{~h}$ dark cycle for 24-48 h. Procedures for Test 1 and Test 2, as well as training, remained the same. Separate sets of larvae were used for each time point as testing larvae (exposing them to the unconditioned stimulus [US] alone) after training will lead to extinction of the conditioned preference.

Pharmacology. Larvae were incubated in each compound exclusively during the $3 \mathrm{~h}$ training period; $1000 \times$ stock solutions were made by dissolving MK-801 (M107; Sigma-Aldrich), puromycin (P8833; SigmaAldrich), or cycloheximide (PS1002; Sigma-Aldrich) in 100\% DMSO (D2650; Sigma-Aldrich) and stored at $-20^{\circ} \mathrm{C}$. Stock solutions were dissolved in E3 embryo medium to achieve the final indicated concentration. After training, larvae were removed from the training chamber and washed briefly before being placed in a 12-well plate as described above.

\section{Results}

This place-conditioning paradigm pairs a social reward, visual access to a group of conspecifics, with a distinct environment indicated by light intensity. We designed two different behavioral chambers (Fig. 1a). Both the testing and the training chamber consisted of 14 channels, each housing an individual larva. In the testing chamber, the barriers between channels were completely opaque, whereas in the training chamber half of each barrier was opaque and the remainder was transparent. This creates two distinct environments: an "isolated" environment in which a larva cannot see its neighbors, and a "social" environment in which the larva has visual access to conspecifics in neighboring channels (Fig. 1a). The testing and training chambers were placed in an enclosed behavioral box that isolated the chambers from any outside visual or acoustic stimuli. The behavioral chamber had a semitransparent bottom, onto which different light environments were projected, and an opening at the top, into which a camera was fixed to monitor larvae position (Fig. 1b).

We first examined the innate preference of larvae $(6-8 \mathrm{dpf})$ for the light and social environment. To test for light preference, $6-8$ dpf wild-type larvae were individually placed in the channels of the testing chamber, which was then illuminated with two different light intensities creating equally sized dark and light environments (Fig. 1c). Larvae position was captured every $10 \mathrm{~s}$ for a $15 \mathrm{~min}$ period. The percentage of time larvae spent in the light environment was determined. Scores $>50 \%$ indicate a preference for the light environment, and scores $<50 \%$ indicate a preference for the dark environment. Social preference was evaluated in a similar manner. Here larvae were placed individually in the training chamber (with an opportunity to view conspecifics), and the entire chamber was illuminated using the settings for either dark or light environment for a $15 \mathrm{~min}$ period (Fig. 1d). Scores $>50 \%$ indicate a preference for the social environment, whereas scores $<50 \%$ indicate a preference for the individual environment.

Larvae of 6-8 dpf showed a moderate preference for light under the conditions tested here, which increased slightly, although not significantly, with age ( $6 \mathrm{dpf}$ vs $8 \mathrm{dpf}, p=0.091$; Fig. 1c). In contrast, larvae showed a strong preference for the social environment (Fig. 1d). Despite a trend for slightly higher scores in the light environment (likely because of better visibility of conspecifics), this social preference remained stable with age and was robust across illumination conditions. The preference for the social environment was dependent on the presence of larvae in the adjacent channels (Fig. 1e), demonstrating that visual access to conspecifics, not other aspects of the social environment, acts as the reward. These results demonstrate that naive larval zebrafish show moderate light environment preference and strong social environment preference, which remains stable across a $3 \mathrm{~d}$ age span.

We next determined whether visual access to conspecifics could serve as an US in an associative learning paradigm, using a simple spatial manipulation of light intensity as a conditioned stimulus (CS) (Fig. 2a). The chamber was illuminated such that half of each channel was "light" relative to the other half ("dark"; see Materials and Methods). As larvae exhibit an innate preference for the light environment, we used "dark" illumination as the CS to increase the dynamic range of preference change after conditioning. The paradigm consisted of three distinct phases (Fig. 2a). During the two 15 min periods of Test 1, the naive light preference of individual zebrafish larvae was determined. This was followed by a $3 \mathrm{~h}$ training period during which the dark (CS) and social (US) environment were paired. After training, the larvae were individually placed overnight in a 12 -well plate, which, in turn, was placed in an incubator with a $10 \mathrm{~h}$ dark $/ 14 \mathrm{~h}$ light cycle. Approximately $14 \mathrm{~h}$ after training, the conditioned light preference of each larval zebrafish was determined (Test 2). Control larvae were exposed to the same procedure, except that this group was placed in the testing chamber, which does not possess a social environment, during training.

After training, the experimental larvae, which were exposed to pairings of the social and dark environment, exhibited a significant shift in preference from the light to the dark environment as seen in the sample behavioral traces (Fig. 2b). This change in preference is quantified in Figure $2 c$, where the percentage of time spent in the light environment before training (Test 1 ) of both paired (black) and control (white) larvae is plotted on the $x$-axis, whereas the percentage of time spent in the light environment after training (Test 2, first $15 \mathrm{~min}$ period) is plotted on the $y$-axis. Whereas control larvae clustered in the upper right quadrant of 


\section{a}

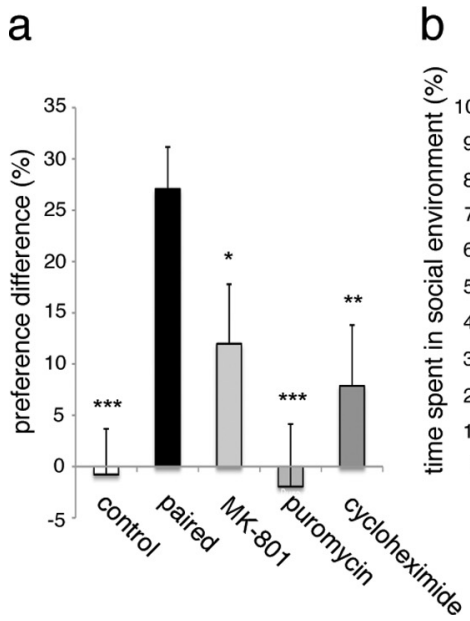

b

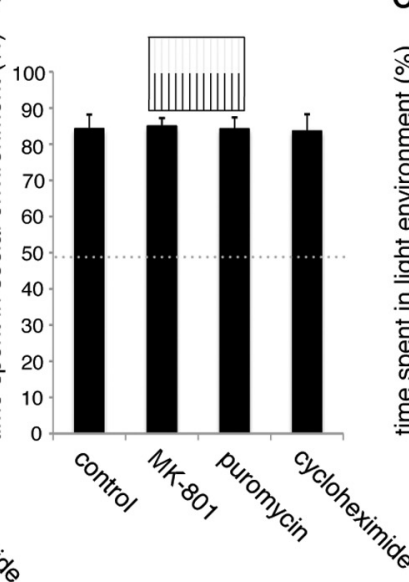

C

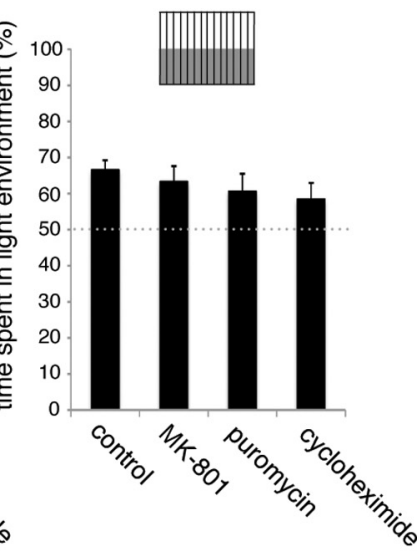

Figure 4. Memory is protein synthesis- and NMDAR-dependent. $\boldsymbol{a}$, Light preference differences of experimental (black), control (white), and larvae exposed to puromycin (gray), cycloheximide (dark gray), or MK-801 (light gray) tested $14 \mathrm{~h}$ after training; positive values indicate a preference for the dark area of the chamber. Error bars indicate SEM. ${ }^{* * *} p<0.001$ and Cohen's $d \geq 0.8$. ${ }^{* *} p<$ 0.01 and Cohen's $d \geq 0.6{ }^{*} p<0.05$ and Cohen's $d \geq 0.45 . n=35-47$ per condition. Percentage of time spent in social (b) and light (c) environment during 15 min period after $3 \mathrm{~h}$ incubation in puromycin $(5 \mu \mathrm{g} / \mathrm{ml})$, cycloheximide $(10 \mu \mathrm{m})$, or MK-801 (100 $\mu \mathrm{m})$. Differences were not statistically significant. Error bars indicate SEM. $n=25-84$ per condition.

the scatter plot, indicating that their preference for light environment remained constant, paired larvae clustered in the lower right quadrant, indicating a preference shift from light to dark environment after training. We quantified the "preference difference" for both experimental and control groups by subtracting the percentage of time spent in the light environment during Test 2 (post-training) from that observed during Test 1 (pretraining) for individual fish (Fig. 2d). Whereas the control group showed no light preference difference, the experimental group exhibited a significant preference difference $\left(p=4.1 \times 10^{-8}\right.$; Cohen's $\mathrm{d}=$ $1.01, r=0.45)$. This indicates that the larvae exposed to dark environment paired with the social reward were able to learn the association between the two stimuli. The distribution of this preference difference centered on zero for the control group, whereas it was significantly shifted in the direction of dark environment preference for the experimental group (Fig. 2e). An unpaired control group, which was exposed to unpaired presentations of light and the social environment, showed no significant preference difference after training (Fig. $2 f$ ). As we did not record behavior during training, we cannot state whether the behavior of one larva during training influenced the behavior of its neighbor during testing after training. However, there was no apparent correlation between the performances of neighbors during testing after training. These results indicate that freely behaving 6-8 dpf zebrafish larvae can exhibit associative learning and memory that can be retained overnight.

Next, to investigate the duration of memory, we varied the length of the interval between the last training session and the memory test (Test 2) from 14 to $48 \mathrm{~h}$. The trained zebrafish larvae showed significant memory retention at intervals up to and including $36 \mathrm{~h}$ (Fig. 3a). Only when the memory interval was increased to $48 \mathrm{~h}$ was the memory no longer significant, demonstrating that the memory was stable for at least $36 \mathrm{~h}$. The preference difference distributions of the 36 and $48 \mathrm{~h}$ interval groups did not show a normal distribution like the other groups (Fig. $3 b$ ) but rather revealed two groups, one with low negative preference difference (indicating loss of memory over time) and the other with a moderate positive preference difference, indicating that a subgroup may be able to retain the associative memory for as long as $48 \mathrm{~h}$. Hence, memory retention in this paradigm is remarkably stable.
The NMDAR has been shown to play a fundamental role in learning and memory and to underlie synaptic plasticity, including LTP and LTD (Riedel et al., 2003). Two paralogs of each of the five mammalian NMDAR subunits are present in zebrafish, and associative learning in adult zebrafish is NMDAR-dependent (Wong et al., 1986; Blank et al., 2009; Sison and Gerlai, 2011). We incubated larvae in the selective noncompetitive NMDAR antagonist MK-801 $(100 \mu \mathrm{M})$ during training. Whereas incubation with MK-801 did not affect the unconditioned light or social preference (Fig. 4b,c), memory retention was significantly impaired compared with the experimental group ( $p=0.027$; Cohen's $\mathrm{d}=0.48, r=0.23$ ), suggesting a requirement for NMDAR activity (Fig. $4 a$ ).

At a mechanistic level, long-term memory can be distinguished from short-term memory by its requirement for new protein synthesis (Davis and Squire, 1984). By applying a PSI during the $3 \mathrm{~h}$ training period, we tested whether the social placeconditioning memory requires new protein synthesis. The addition of puromycin $(5 \mu \mathrm{g} / \mathrm{ml})$, which has been demonstrated to inhibit protein synthesis in larval zebrafish (Hinz et al., 2012), to the embryo medium during training completely abolished memory formation when tested overnight. A different PSI (cycloheximide; $10 \mu \mathrm{M}$ ) had less profound but still significant effect on learning (Fig. 4a). We did not observe obvious differences in overall larval movement with drug exposure, nor did social or light environment preferences change in the presence of PSIs (Fig. $4 b, c$ ). These data show that the associative long-term memory exhibited by the larval zebrafish requires new protein translation.

\section{Discussion}

In this study, we have described a new associative placeconditioning paradigm for larval zebrafish. During training, experimental larvae learned to associate the social reward of visual access to a group of conspecifics with a dark environment. In contrast, control groups that were either not exposed to the social reward or for whom the social reward and light environment were presented in an unpaired manner, did not change their preference for light environment. Furthermore, we demonstrated that this associative memory was remarkably stable, lasting for up to $36 \mathrm{~h}$. Incubating larvae in protein synthesis 
inhibitors or NMDAR antagonists during training prevented or impaired memory formation, respectively, confirming that this associative learning is protein synthesis and NMDAR-dependent.

Here we demonstrate that 6-8 dpf larvae are capable of associative learning and that the unconditioned stimulus of visual access to a group of conspecifics can act as a social reward. The paradigm described is one of the few to induce long-term memory formation in a juvenile of any species and may therefore provide important insights into the development of learning in vertebrates. The zebrafish is a social species known to aggregate, and visual access of conspecifics has previously been described to have rewarding properties in adult zebrafish (Al-Imari and Gerlai, 2008; Gómez-Laplaza and Gerlai, 2010; Sison and Gerlai, 2011). Little is known, however, about the natural ecology of the larval zebrafish. We show here that they prefer to aggregate with conspecifics at an early age, implying that such behaviors may be ecologically relevant.

As this paradigm is simple and does not involve restraining of larvae, it can easily be serialized for high-throughput behavioral or pharmacological screens or proteomic approaches. Furthermore, as the formation of the long-term associative memory is protein synthesis-dependent, this paradigm may be ideally suited to be paired with FUNCAT and BONCAT techniques (Dieterich et al., 2006, 2010; Hinz et al., 2012) to visualize cells or neuronal circuits underlying memory formation, as well as to identify proteins differentially translated during memory formation.

\section{References}

Agranoff BW, Klinger PD (1964) Puromycin effect on memory fixation in the goldfish. Science 146:952-953. CrossRef Medline

Aizenberg M, Schuman EM (2011) Cerebellar-dependent learning in larval zebrafish. J Neurosci 24:8708-8712. CrossRef Medline

Al-Imari L, Gerlai R (2008) Sight of conspecifics as reward in associative learning in zebrafish (Danio rerio). Behav Brain Res 189:216-219. CrossRef Medline

Best JD, Berghmans S, Hunt JJ, Clarke SC, Fleming A, Goldsmith P, Roach AG (2008) Non-associative learning in larval zebrafish. Neuropsychopharmacology 33:1206-1215. CrossRef Medline

Blank M, Guerim LD, Cordeiro RF, Vianna MR (2009) A one-trial inhibitory avoidance task to zebrafish: rapid acquisition of an NMDAdependent long-term memory. Neurobiol Learn Mem 92:529-534. CrossRef Medline

Braubach OR, Wood HD, Gadbois S, Fine A, Croll RP (2009) Olfactory conditioning in the zebrafish (Danio rerio). Behav Brain Res 198:190198. CrossRef Medline

Davis HP, Squire LR (1984) Protein synthesis and memory: a review. Psychol Bull 96:518-559. CrossRef Medline

Del Bene F, Wyart C, Robles E, Tran A, Looger L, Scott EK, Isacoff EY, Baier $\mathrm{H}$ (2010) Filtering of visual information in the tectum by an identified neural circuit. Science 330:669-673. CrossRef Medline

Dieterich DC, Link AJ, Graumann J, Tirrell DA, Schuman EM (2006) Selective identification of newly synthesized proteins in mammalian cells using bioorthogonal noncanonical amino acid tagging (BONCAT). Proc Natl Acad Sci U S A 103:9482-9487. CrossRef Medline

Dieterich DC, Hodas JJ, Gouzer G, Shadrin IY, Ngo JT, Triller A, Tirrell DA, Schuman EM (2010) In situ visualization and dynamics of newly synthesized proteins in rat hippocampal neurons. Nat Neurosci 7:897-905. CrossRef Medline

Fetcho JR, McLean DL (2010) Some principles of organization of spinal neurons underlying locomotion in zebrafish and their implications. Ann N Y Acad Sci 1198:94-104. CrossRef Medline

Gómez-Laplaza LM, Gerlai R (2010) Latent learning in zebrafish (Danio rerio). Behav Brain Res 208:509-515. CrossRef Medline

Hinz FI, Dieterich DC, Tirrell DA, Schuman EM (2012) Non-canonical amino acid labeling in vivo to visualize and affinity purify newly synthesized proteins in larval zebrafish. ACS Chem Neurosci 3:40-49. CrossRef Medline

Lee A, Mathuru AS, Teh C, Kibat C, Korzh V, Penney TB, Jesuthasan S (2010) The habenula prevents helpless behavior in larval zebrafish. Curr Biol 20:2211-2216. CrossRef Medline

Mathur P, Lau B, Guo S (2011) Conditioned place preference behavior in zebrafish. Nat Protoc 6:338-345. CrossRef Medline

Riedel G, Platt B, Micheau J (2003) Glutamate receptor function in learning and memory. Behav Brain Res 140:1-47. CrossRef Medline

Roberts AC, Reichl J, Song MY, Dearinger AD, Moridzadeh N, Lu ED, Pearce K, Esdin J, Glanzman DL (2011) Habituation of the C-start response in larval zebrafish exhibits several distinct phases and sensitivity to NMDA receptor blockade. PLoS One 6:e29132. CrossRef Medline

Sison M, Gerlai R (2011) Associative learning in zebrafish (Danio rerio) in the plus maze. Behav Brain Res 207:99-104. CrossRef Medline

Wolman MA, Jain RA, Liss L, Granato M (2011) Chemical modulation of memory formation in larval zebrafish. Proc Natl Acad Sci U S A 108: 15468-15473. CrossRef Medline

Wong EH, Kemp JA, Priestley T, Knight AR, Woodruff GN, Iversen LL (1986) The anticonvulsant MK- 801 is a potent $N$-methyl-D-aspartate antagonist. Proc Natl Acad Sci U S A 83:7104-7108. CrossRef Medline

Wyart C, Del Bene F, Warp E, Scott EK, Trauner D, Baier H, Isacoff EY (2009) Optogenetic dissection of a behavioural module in the vertebrate spinal cord. Nature 461:407-410. CrossRef Medline 